\title{
A Stable Controller Design with Fuzzy Rules in Accordance with Experts' Knowledge
}

\section{Hugang HAN*1, Hiroko KAWABATA*2, Shuta MURAKAMI*3}

\begin{abstract}
Although innumerable research has challenged the design of fuzzy control systems from the standpoint of stability, most of them are just considering the fuzzy rules as an approximator to deal with the unknown functions in the plant to be controlled. Also, the fuzzy rules are changed completely from their origins which are obtained based on the views of experts after the parameters in the fuzzy rules are tuned. Actually, the primary idea of fuzzy control is to employ the knowledge of experts to control a plant instead of the algorithm extracted from the mathematical model of the plant. Clearly, if the original fuzzy rules are changed at all, what is the point of fuzzy control? In this paper, we consider a few cases to show how to develop a stable control system which persistently maintains the fuzzy rules in accordance with experts' views. Also, computer simulations will support the approach appeared in this paper.
\end{abstract}

Keywords: Fuzzy control, system stability, experts' knowledge

\section{INTRODUCTION}

For the last decade or more, a large quantity of research has focused on the adaptive fuzzy control systems, and has achieved success in a sense (e.g. [1]-[5] and references therein). In order to develop such an adaptive fuzzy control system, one of the important approaches was to adopt the Lyapunov synthesis approach, and to consider the fuzzy rules involved in the approach as some approximators to deal with the unknown parts (functions) in the plant to be controlled [6]. Furthermore, the classic adaptive control theory has been merged to compose the update law of parameters involved in the system including the fuzzy rules in the view of "stability" of system to be concerned [1].

Herein let's consider a question: "What is the initial purpose for using fuzzy control?" One, of the key reasons is that, instead of the computational

* 1 School of Business, Hiroshima Prefectural University

*2 Faculty of Economics, Daiichi Keizai University

*3 Faculty of Engineering, Kyushu Institute of Technology controllers like a PID obtained from the mathematical models of the plants to be controlled, the control strategy is described in the human-being-oriented form (i.e., if-then rule) obtained by experienced experts. In other words, experts can take reasonable control actions, even in the time-varying conditions of the process, their nonlinearities, and existing disturbances. This means that knowledge about the plant and the control process is well represented in the if-then rules.

Apparently, in an adaptive fuzzy control system, if the fuzzy rules are only considered as the (fuzzy) approximators and finally their parameters are tuned completely differently from their original faces, then there are two questions that arise: (1) Why fuzzy rules? If we just want to approximate some unknown functions in the plant, some other soft computing methods like neural networks seem more attractive; and (2) Why fuzzy control? If the experienced fuzzy rules are almost all changed in the control process, where is the knowledge of experts going? Moreover, we should note that in the 
case of (1), we tune the parameters in the fuzzy approximators only from the standpoint of system stability, and as a result, the fuzzy approximators, in most cases, are not real approximators of their corresponding functions at all, because the errors between the both are sometimes very huge. Therefore, once we say fuzzy control, it seems that we should keep the original fuzzy rules. In this way, however, how to maintain the stability of the whole control system is a question we have to answer.

In this paper, we consider a few cases to show how to develop a stable fuzzy control system where the experts' know-how about the plant and the control process is utilized wherever possible. Also, computer simulations will support the approach appeared in this paper.

\section{PROBLEM STATEMENT}

This paper focuses on the design of adaptive fuzzy control algorithms for a class of nonlinear systems whose equation of motion can be expressed in the canonical form:

$$
x^{(n)}=f(X(t))+b(X(t)) u(t)
$$

where $X^{T}(t)=\left[x(t), \dot{x}(t), \ldots, x^{(n-1)}(t)\right]$ is the state vector, $u(t)$ is the control input, $f$ is an unknown linear or nonlinear function of $X$, and $b$ is the control gain. It should be noted that more general classes of nonlinear systems could be transformed into this structure [9]. For the sake of simplicity throughout the remainder of this paper, we will omit time $t$ unless a confusion arises.

The control objective is to force the output $x$ to follow a specified desired trajectory. Let $x_{d}$ be the desired trajectory and define the tracking error,

$$
\tilde{x}=x-x_{d}
$$

The problem is thus to design a controller $u$ for (1) which ensures that $\tilde{x} \rightarrow 0$ as $t \rightarrow \infty$ while maintaining the stability of all signals involved in the system. Here in this paper, we take $b=1$ for the sake of stressing a clear design procedure.

Now, we adopt the variable structure theory to construct our adaptive fuzzy control system. The sliding mode hyperplane is firstly defined as

$$
s=\left(\frac{d}{d t}+\lambda\right)^{n-1} \tilde{x} \quad \text { with } \lambda>0
$$

where $\lambda$ defines the bandwidth of the error dynamics of the system. The equation defines a time -varying hyperplane in $R^{n}$ on which the tracking error $\tilde{x}$ decays exponentially to zero, so that perfect tracking can be asymptotically obtained by maintaining this condition. In this case, the control objective becomes the design of controller to force $s$ $\rightarrow 0$ as $t \rightarrow \infty$.

The time derivative of the error metric can be written as

$$
\dot{s}=f+u-x_{d}^{(n)}+\Lambda^{T} \tilde{X}
$$

where $\Lambda^{T}=\left[0, \lambda^{n-1},(n-1) \lambda^{n-2}, \ldots,(n-1) \lambda\right], \tilde{X}^{T}=$ $\left[\tilde{x}, \dot{\tilde{x}}, \ldots, \tilde{x}^{(n-1)}\right]$. Referring to system (1), it naturally suggests that when $f$ is known, a controller of form,

$$
u=-k s-f+x_{d}^{(n)}-\Lambda^{\tau} \tilde{X}
$$

leads to $\dot{s} s=-k s^{2}$, and hence, $\tilde{x} \rightarrow 0$ as $t \rightarrow \infty$, where $k>0$. However, as mentioned, the function $f$ is unavailable. Therefore, the problem is how $u$ can be determined when a system involves such an unknown function. Here, let's classify what cases can exist in such a system before we determine the controller. Generally, there are three cases: the first case is that we do not have any information available about the functions so that we need to approximate them from scratch; the second case is that we already have good knowledge about the functions, which is referred to as a well-tuned-fuzzy-approximator in this paper; and the third case is that there is a so-called well-tuned-fuzzy-controller given by experts about the control process. For the latter two cases, the knowledge about the functions and the control process, clearly, should be utilized as much as possible in the development of the control system. In the next section, we will give details to show how to develop a stable controller in the three cases, but firstly, the fuzzy system will be reviewed briefly.

\section{STABLE FUZZY \\ CONTROLLERS}

\subsection{Fuzzy approximator}

Genarally, a fuzzy system consists of three main 
parts: the rule base, the inference engine module, and the difuzzification model. The rule base, also known as knowledge base, stores the rules, which contain the qualitative and heuristic knowledge, in the form of a collection of fuzzy IF antecedent THEN consequent rules that can be interpreted form a point of view of automatic control as IF condition THEN action. Herein, we consider a subset $U \in R^{n}$ of a fuzzy system with singleton consequent, product inference, and Gaussian membership function. Hence, such a fuzzy system can be written as

$$
\mathcal{F}(X)=W^{T} \cdot G(X)
$$

where $X^{T}=\left[x_{1}, x_{2}, \ldots, x_{n}\right], W^{T}=\left[\omega_{1}, \omega_{2}, \ldots, \omega_{N}\right]$ with $\omega_{j}$ being the so-called connection weight; $G^{T}(X)=$ $\left[g_{1}(X), g_{2}(X), \ldots, g_{N}(X)\right]$, and $g_{j}(X)=$

$\frac{\prod_{i=1}^{n} \mu_{A_{j}^{i}\left(x_{i}\right)}}{\sum_{j=1}^{N} \prod_{i=1}^{n} \mu_{A_{j}^{i}\left(x_{i}\right)}}$ where $\mu_{A_{j}^{i\left(x_{i}\right)}}$ is a Gaussian member. ship function, defined by

$$
\mu_{A j}^{i\left(x_{i}\right)}=\exp \left[-\left(\frac{x_{i}-\xi_{j}^{i}}{\sigma_{j}^{i}}\right)^{2}\right]
$$

where $\xi_{j}^{i}$ indicates the position, and $\sigma_{j}^{i}$ indicates the variance of the membership function.

We now can show an important property on the above fuzzy system [6].

Theorem 1 For any given real continuous function $f$ on the compact set $U \in R^{n}$ and arbitrary $\varepsilon^{*}$, there exists an optimal fuzzy system expansion $\mathcal{F}^{*}(X)=$ $W^{* T} \cdot G(X)$ with, such that

$$
\sup _{X \in U}\left|f(X)-\mathcal{F}^{*}(X)\right|<\varepsilon^{*}
$$

where,

$$
W^{*}=\arg \min _{W \in \Omega \subset R^{n}}\left\{\sup _{X \in U \subset R^{n}}\left|f(X)-W^{T} \cdot G(X)\right|\right\}
$$

The theorem above shows that the fuzzy system $\mathcal{F}$ can be viewed as an approximator to approximate a real continuous function $f$. In this paper such an approximator is referred to be as a fuzzy approximator.

\subsection{No knowledge available}

It is an obstacle to have such an unknown function $f$ on the process of system development. In order to deal with it, one way is to approximate the unknown functions. Thinking of the approximator, a lot of literature employ the fuzzy approximator as shown in the previous subsection 3.1. Here we adopt this idea for the time being. Let us denote $f^{*}(X)=$ $W_{f}^{* T} G_{f}(X)$ to be the fuzzy approximator of the unknown function $f$. Then, there is a small positive value $\varepsilon_{f}^{*}$ such that, the error,

$$
\varepsilon_{f}=f-f^{*}
$$

which is referred to as reconstruction error, satisfies the following inequality,

$$
\left|\varepsilon_{f}\right|<\varepsilon_{f}^{*}
$$

Apparently, the optimal vector $W_{f}^{*}$ is unknown while $G_{f}(X)$ is an available vector function in the fuzzy approximator $f^{*}(X)=W_{f}^{* T} G_{f}(X)$, therefore, as usual, its estimate, denoted as $\hat{f}(X)=\hat{W}_{f}^{T} G_{f}(X)$ is adopted, and will be tuned based on the error dynamics $s$.

Now, we are ready to develop our control system. Inspired by the above control structure in (5), our controller is now described as

$$
u=u_{f d}+u_{e t}+u_{e c}
$$

where $u_{f d}$ is a linear feedback component described as

$$
u_{f d}=x_{d}^{(n)}-\Lambda^{T} \bar{X}-k s
$$

$u_{e t}$ is an estimate component for the unknown function $f$ and described as

$$
u_{e t}=-\hat{f}=-\hat{W}_{f}^{T} G_{f}(X)
$$

where $\hat{W}_{f}$ is a tunable parameter vector and tuned by adaptive law,

$$
\dot{\hat{W}}_{f}=\Gamma_{f} G_{f}(X) s
$$

where $\hat{W}_{f}$ is the estimate of $W_{f}^{*}$, and $\Gamma_{f}$ is an appropriate symmetric positive definite matrix which determines the adaptation rate. And $u_{e c}$ is a complement component, which complements the error between $f$ and $\hat{f}$, described as

$$
u_{e c}=-\hat{\varepsilon}_{f} \operatorname{sgn}(s)
$$

where $\hat{\varepsilon}_{f}$ is the estimate of $\varepsilon_{f}^{*} \cdot \hat{\varepsilon}_{f}$ also has a purpose of avoiding a prior knowledge about the reconstruction error. $\hat{\varepsilon}_{f}$ is estimated by,

$$
\dot{\dot{\varepsilon} f}=\gamma_{f}|s|
$$

where $\gamma_{f}$ is the adaptation rate. A block diagram of 
this controller structure is depicted in Fig. 1, where the block labeled "Filter" is an operation system which generates $x_{n}^{(n)}, \tilde{X}$, and $s$ form signals $x_{d}$, and $x$.

We start the system stability analysis by defining the Lyapunov function candidate:

$$
V=\frac{1}{2}\left(s^{2}+\tilde{W}_{f} \Gamma_{f}^{-1} \tilde{W}_{f}+\gamma_{f} \tilde{\varepsilon} f_{f}^{2}\right)
$$

where,

$$
\begin{aligned}
& \tilde{W}_{f}=W_{f}-W_{f}^{*} \\
& \tilde{\varepsilon}_{f}=\hat{\varepsilon}_{f}-\varepsilon_{f}^{*}
\end{aligned}
$$

From (4), (9) and (11), s can be rewritten as

$$
\begin{aligned}
\dot{s} & =-k s+f-\hat{f}-\hat{\varepsilon}_{f} \operatorname{sgn}(s) \\
& =-k s+\varepsilon_{f}+f^{*}-\hat{f}--\hat{\varepsilon}_{f} \operatorname{sgn}(s) \\
& =-k s+\tilde{f}+\varepsilon_{f}-\hat{\varepsilon}_{f} \operatorname{sgn}(s)
\end{aligned}
$$

Taking the derivative of both sides of (17), and substituting (14), (16), and (20) into it, we have

$$
\begin{aligned}
\dot{V} & =s \dot{s}+\tilde{W}_{f}^{T} \Gamma_{f}^{-1} \hat{W}_{f}+\gamma_{f}^{-1} \tilde{\varepsilon}_{f} \hat{\varepsilon}_{f} \\
& =-k s^{2}+\tilde{f}_{S}+\varepsilon_{f} s-\hat{\varepsilon}_{f}|s|-\tilde{\varepsilon}_{f} s \\
& \leq-k s^{2}+\left|\varepsilon_{f}\right||s|-\hat{\varepsilon}_{f}|s|-\tilde{\varepsilon}_{f} s \\
& \leq-k s^{2}
\end{aligned}
$$

It means all signals $\left(s, \hat{W}_{f}, \hat{\varepsilon}_{f}\right)$ are bounded, and $s \rightarrow$ 0 as $t \rightarrow \infty$; Furthermore, tracking error $\tilde{x} \rightarrow 0$ as $t$ $\rightarrow \infty$.

Q.E.D.

Remark 1 It should be noted that the bound of $\varepsilon_{f}^{*}$ does not need to be known in the approach above. In this way, in the approximation of $f$ the necessity to assume a prior knowledge of the bound on the reconstruction error can be removed. Of course, if the bound $\varepsilon_{f}^{*}$ is given,

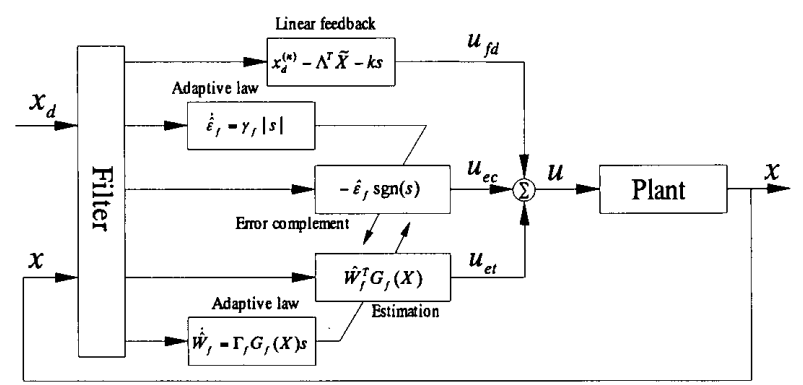

Figure 1: Closed-loop control system without knowledge available then the complement component (15) can be replaced by

$u_{e c}=-\varepsilon_{f}^{*} \operatorname{sgn}(s)$

Consequently, the estimate $\hat{\varepsilon}_{f}(16)$ is no longer needed, and stability is surely guaranteed.

Remark 2 The parameters in the fuzzy approximator are completely tuned by adaptive law (14) only based on the needs of the system stability. In other words, the fuzzy approximator does not involve any knowledge from the experts, and consequently the approximateness is sometimes very poor though the control performance is good. Therefore, strictly speaking, in our view the control system, even if it involves fuzzy rules, is no longer a fuzzy control system, but an adaptive control system.

\subsection{Well-tuned-fuzzy-approximator}

When we try to develop a controller for system (1), somehow we have to deal with the unknown function $f$. The second case is that some experts have enough knowledge about the unknown function. It means the experts can relatively accurately describe the function by fuzzy rules. Therefore, in this case the well-tuned-fuzzy-approximator should be utilized as much as possible. In other words, we do not need to approximate the unknown function from scratch at all.

Here in this subsection, suppose that we have the well-tuned-fuzzy-approximator, in symbol $\mathcal{F}$, for the unknown function $f$. Let $\varepsilon_{f}$ be the error between $f$ and $\mathcal{F}$ as follows.

$$
\varepsilon_{f}=f-\mathcal{F}
$$

which is referred to as approximator error. It is reasonable to suppose that there exists a small positive value $\varepsilon_{f}^{*}$ as well so that

$$
\left|\varepsilon_{f}\right| \leq \varepsilon_{f}^{*}
$$

and referring to the controller in the previous subsection, it is natural to compose the controller in this case as

$$
u=u_{f d}+u_{f a}+u_{e c}
$$

where $u_{f d}$, and $u_{e c}$ are the same as (12), and (15), 
respectively, and $u_{f a}$ is the well-tuned-fuzzy-approximator $\mathcal{F}$ :

$$
u_{f a}=-\mathcal{F}
$$

Clearly, the parameters in $\mathcal{F}$ do not need to be tuned at all. A block diagram of this controller structure is depicted in Fig. 2.

The control system stability analysis can be done in the same way as in the previous subsection 3.2 .

Remark 3 As an approximator of an unknown function, $\mathcal{F}$ is not just being described in the form of fuzzy rules, but also in other forms like neural networks. If $\mathcal{F}$ is not a fuzzy approx. imator, then the system above is not called a fuzzy control system any more. Actually, it seems that there are only a few cases where we can get the fuzzy approximators for the specific functions in the control system.

\subsection{Well-tuned-fuzzy-controller}

Generally, a fuzzy control system can be depicted as Fig. 3 where the block Filter generates input signals like tracking error or its difference from $x_{d}$, and $x$, and the block $\mathcal{F C}$ represents the fuzzy controller, which generates control moments $u$ to the plant from all the input signals.

In quite a few cases, experts or operators may have very good knowledge on the control process in terms of fuzzy rules, i.e., well-tuned-fuzzy-controller, to effectively control a specific plant. Therefore, the well-tuned-fuzzy-controller should be involved in the development of the control system as much as possible if it is available.

Here, in this subsection, suppose that we have the well-tuned-fuzzy-controller $\mathcal{F C}$. From (5), the

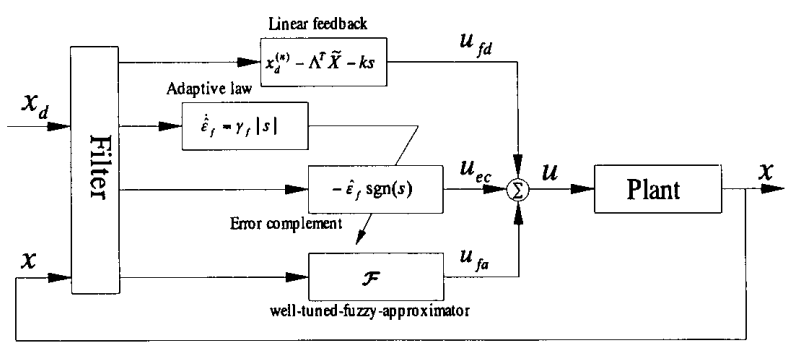

Figure 2: Closed-loop control system with the well-tuned-fuzzy-approximator

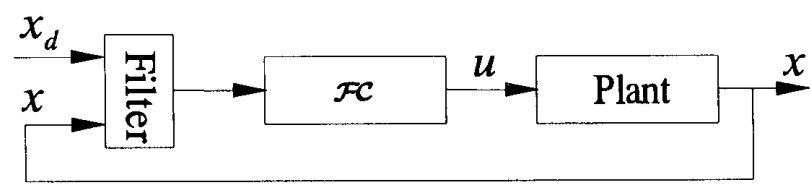

Figure 3: The general fuzzy control system

controller is composed of $-k s-f+x_{d}^{(n)}-\Lambda^{T} \tilde{X}$ (三 $u_{T}$ ), and here the control input (Fig. 3) is determined by $\mathcal{F C}\left(\equiv u_{\mathcal{F}}\right)$. This means $u \mathcal{F}$ is a kind of description (approximation) of $u_{T}$, and not just for the unknown function $f$. Define the error between $u_{t}$ and $u_{t}$ as follows:

$$
\varepsilon u=u_{\mathcal{F}}-u_{T}
$$

Because $u \mathcal{F}$ can effectively control the plant as well, it is reasonable to suppose that there exists a positive value $\varepsilon_{u}^{*}$ so that

$$
\left|\varepsilon_{u}\right| \leq \varepsilon_{u}^{*}
$$

Referring to (24), the controller involving the welltuned-fuzzy-controller $\mathcal{F C}$ is composed of

$$
u=u_{f d}+u_{\mathcal{F}}+u_{e c}
$$

where $u_{f d}$ is the linear feedback component described as

$$
u_{f d}=-\varkappa s, \quad \varkappa>0
$$

And $u_{e c}$ is the complement component, which complements the error between $u \mathcal{F}$ and $u_{T}$, described as

$$
u_{e c}=-\hat{\varepsilon} u \operatorname{sgn}(s)
$$

where $\hat{\varepsilon}_{u}$ is the estimate of $\varepsilon_{u}^{*}$ that is estimated by,

$$
\dot{\hat{\varepsilon} u}=\gamma_{u}|s|
$$

where $\gamma_{u}$ is the adaptation rate. A block diagram of this controller structure is depicted in Fig. 4.

The stability of the closed-loop system described by (1) - (3), and (28)-(31) is established in the following theorem. The proof is similar to the stability analysis in subsection 3.2 and omitted here because of the shortage of space.

Theorem 2 If the plant (1) is controlled by (28)(30) with the adoptive law (31), then tracking error (2) will be shrunk to zero while maintaining all signals involved in the system bounded. 


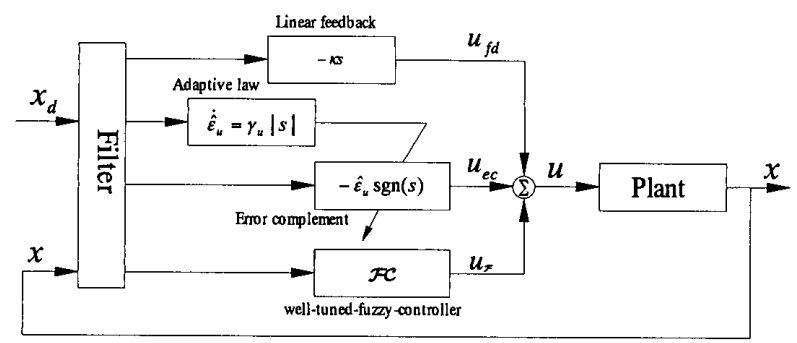

Figure 4: Closed-loop control system with the well-tuned-fuzzy-controller

Remark 4 At first glance, Figs. 2 and 4 have the same structure. However, there are two points that deserve our attention. The first one is that the linear feedback component $u_{f d}$ in Fig. 4 consists only of $s$ while combining with $s, x_{d}^{(n)}$, and $\tilde{X}$ in Fig. 2, in which $x_{d}^{(n)}$, and $\tilde{X}$ are included in $\mathcal{F C}$ in Fig. 4. The second one is that $\mathcal{F C}$ in Fig. 4 is an independent controller, which can effectively control the plant in practice, and $u_{f d}$, and $u_{e c}$ are just auxiliary components in case of system stability being threatened, while $\mathcal{F}$ in Fig. 2 is just an estimator, which deals with the unknown function $f$ in the plant.

\section{SIMULATION STUDY}

To illustrate and clarify the proposed design procedures, we apply them developed in the previous section to control a nonlinear system:

$$
\dot{x}(t)=\frac{1-e^{-x(t)}}{1+e^{-x(t)}}+u(t)
$$

Without the control, i.e., $u(t)=0$, it can be easily seen that the system is unstable, beacuse of $f=$ $\frac{1-e^{-x(t)}}{1+e^{-x(t)}}>0$ for $x(t)>0$, and $f=\frac{1-e^{-x(t)}}{1+e^{-x(t)}}<0$ for $x$ $(t)<0$. The control objective is to force the system state $x(t)$ to the origin, i.e., $x_{d}(t)=0$.

First, let's consider that we do not have any knowledge about the function $f=\frac{1-e^{-x(t)}}{1+e^{-x(t)}}$, and the control process. Therefore, we have to approximate the unknown function $f$ from scratch. In this simulation, the fuzzy approximator is conducted with seven fuzzy rules:

$$
R_{j}: \text { IF } x \text { is } A_{j} \text { THEN } f \text { is } w_{j}
$$

where $j(=1,2, \ldots, 7)$ is rule's number, $A_{j}$ is a fuzzy set that is characterized by fuzzy membership func-

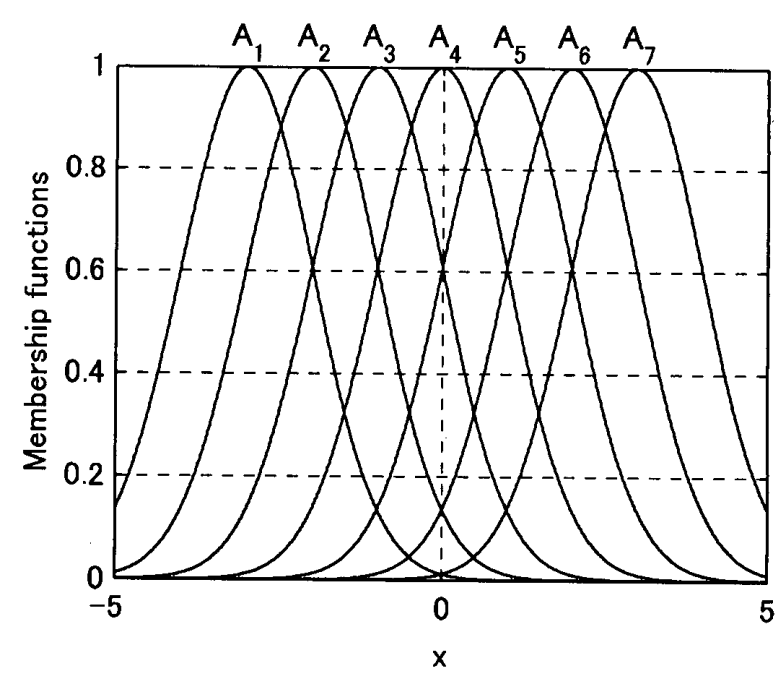

Figure 5: Membership functions in the antecedents of fuzzy rules

tion $\mu_{A j}(x)=\exp \left[-\frac{\left(x-\xi_{j}\right)^{2}}{2}\right]$ with $\xi .=-3,-2,-1$, $0,1,2,3$ for $j=1,2, \ldots, 7$, respectively (Fig. 5), and $w_{j}$ is a singleton value. Because we do not have any knowledge about $f$, we are supposed not to give some appropriate values about $w_{j}$. Therefore, the initial values for $w_{j}(j=1,2, \ldots, 7)$ are just assigned to be value 0.5 uniformly. Then for the needs of system stability, $w_{j}$ are tuned by the adaptive law (14) where $\Gamma_{f}=0.1 I$ with $I$ being an appropriate identity symmetric matrix. The feedback component $u_{f d}$ is calculated by (12) where $k=0.1$. To determine the complement component $u_{e c}$, (16) is used where $\gamma_{f}=0.2$.

The control results are shown in Figs. 6 and 7 where Fig. 6 shows the state's evolution and Fig. 7 shows the amount of the control input $u$. Clearly, the great control performance is observed, even though we do not have any knowledge about the unknown function $f$ and estimate it from scratch. However, because we tune the parameters $w_{j}(j=1$, $2, \ldots, 7)$ only based on the standpoint of the "stability", and not from the viwepoint of "approximateness", the performance of estimator, as shown in Fig. 8, is quite poor. Obviously, the error between $f$ and its fuzzy estimate $\hat{f}$ is covered by the linear feedback component $u_{f d}$ and the complement component $u_{e c}$.

Next, suppose we have the following well-tunedfuzzy-approximator for the unknown function $f$ :

$$
R_{1}: I F x \text { is } A_{1} \text { THEN } f \text { is } 1.02
$$




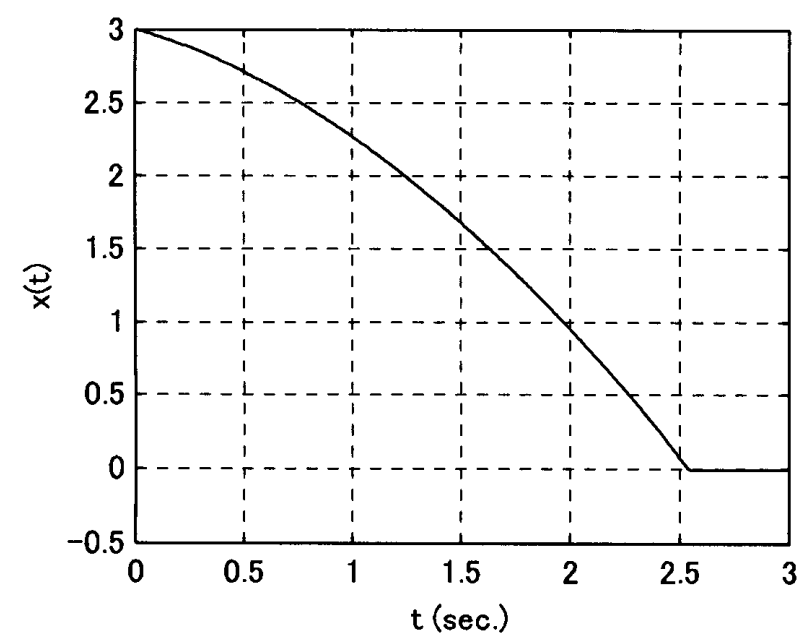

Figure 6: State evolution in the case that has no knowledge available

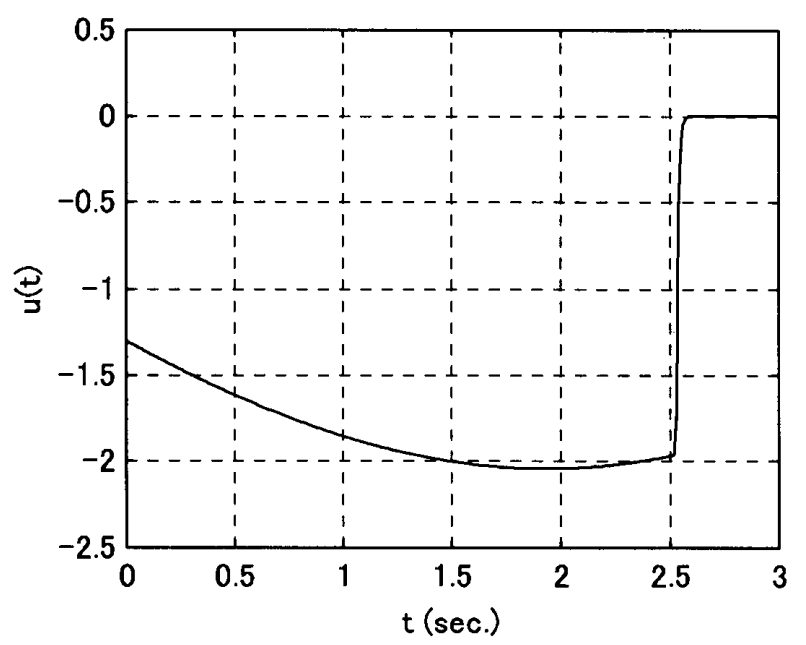

Figure 7: Control moments in the case that has no knowledge available

$\begin{array}{ll}R_{2}: I F x \text { is } A_{2} & \text { THEN } f \text { is } 0.85 \\ R_{3}: I F x \text { is } A_{3} & \text { THEN } f \text { is } 0.54 \\ R_{4}: I F x \text { is } A_{4} & \text { THEN } f \text { is } 0 \\ R_{5}: I F x \text { is } A_{5} & \text { THEN } f \text { is }-0.54 \\ R_{6}: I F x \text { is } A_{6} & \text { THEN } f \text { is }-0.85 \\ R_{7}: I F x \text { is } A_{7} & \text { THEN } f \text { is }-1.02\end{array}$

where the membership function $\mu_{A j}(j=1,2, \ldots, 7)$ are the same as shown in Fig. 5 . The performace of the well-tuned-fuzzy-approximator is shown in Fig. 9 where the solid line is the real function $f$ and the dashed line is the outputs of the well-tuned-fuzzyapproximator during $-3 \leq \mathrm{x} \leq 3$. Involving this fuzzy approximator, the plant (32) in controlled by (24), (25), (12), (15), and (16) where the relevant coefficients such as $\kappa, \gamma_{f}$ are the same as above. The

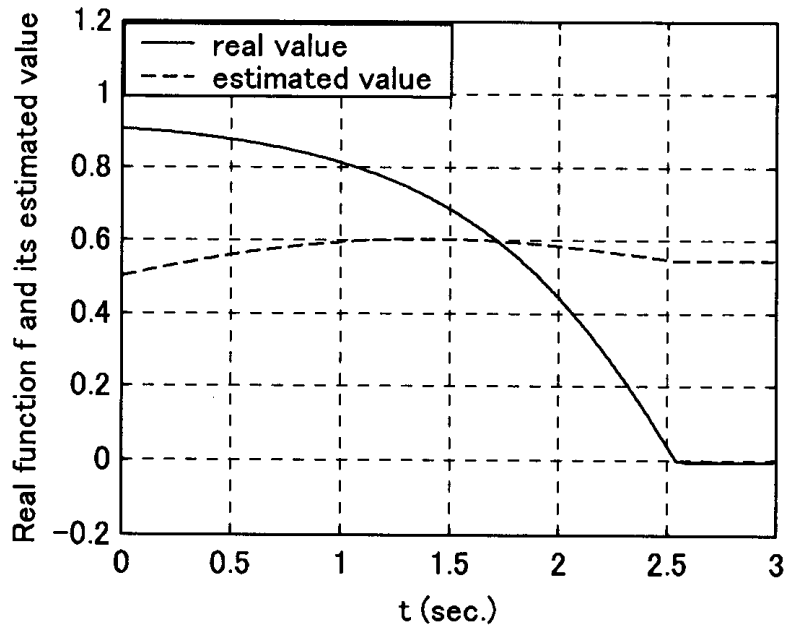

Figure 8: Function $f$ and its estimated value

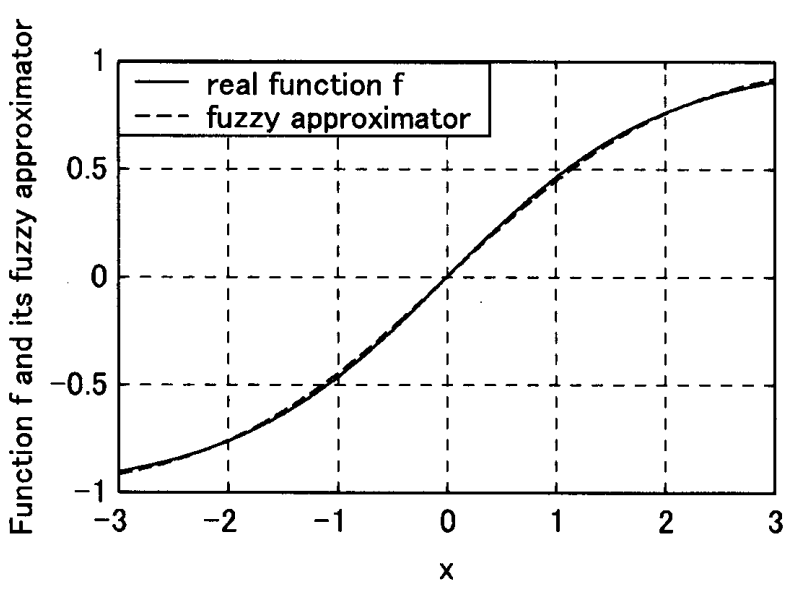

Figure 9: Function $f$ and its fuzzy approximator

control results are shown in Figs. 10 and 11. In comparison with Figs. 6 and 7, apparently, the performance is almost same with the case that the unknown function $f$ estimated by the fuzzy approximator via tuning its parameters by the adaptive law. This means, in a control system, if there are some experts' knowledge about the system such as the well-tuned-fuzzy-approximator, we should employ them as much as possible, and consequently, the control performance is the same with the traditional adaptive control approach, also the control system structure becomes relatively simple.

Lastly, let us consider there is a well-tuned-fuzzy -controller as follows:

$$
R_{i j}: \text { IF } \tilde{x} \text { is } A_{i}, \Delta \tilde{x} \text { is } B_{j} \text { THEN } u \text { is } w_{i j}
$$
where, $\tilde{x}=x-x_{d} ; \Delta \tilde{x}=\tilde{x}-\tilde{x}_{0}$ with $\tilde{x}_{0}$ being the tracking error in the previous sampling time. $A_{i}$ 's mem- 


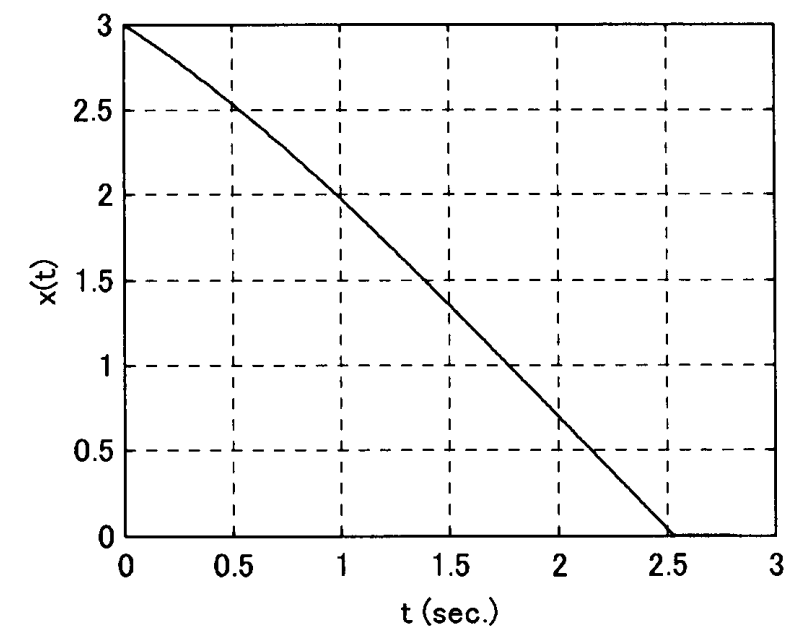

Figure 10: State evolution driven by the controller including the well-tuned-fuzzy-approximator

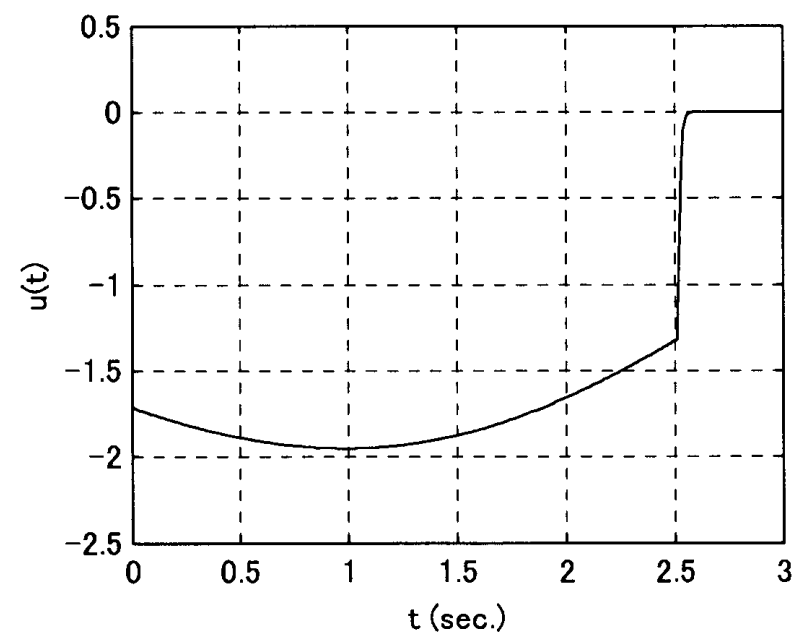

Figure 11: Controller moments including the well -tuned-fuzzy-approximator

bership functions are the same as shown in Fig. 5. For $B_{j}$ 's, their fuzzy membership functions are characterized by $\mu_{B j}(x)=\exp \left[-\frac{\left(x-\xi_{j}\right)^{2}}{2}\right]$ with $\xi .=$ $-1,-0.6,-0.3,0,0.3,0.6,1$ for $j=1,2, \ldots, 7$, respectively. $w_{i j}$ 's are given by Tab.1. The control performance driven by this well-tuned-fuzzy-controller are shown in Figs. 12 and 13. Considered of performances obtained in other cases such as Figs. 6, 7, 9, and 10 , it is reasonable to think highly of the achievement in this case.

Now, using this well-tuned-fuzzy-controller, the plant (32) is controlled by (28) $-(31)$ where $\kappa=0.1$, and $\gamma_{u}=0.2$. Figs. 14 and 15 depict the performance. In comparison with the other cases above, this case,
Table 1 The experts' fuzzy controller

\begin{tabular}{|c|c|c|c|c|c|c|c|c|}
\hline \multicolumn{2}{|c|}{} & \multicolumn{7}{|c|}{$\Delta \tilde{x}$} \\
\cline { 2 - 9 } \multicolumn{1}{|c|}{} & $B_{1}$ & $B_{2}$ & $B_{3}$ & $B_{4}$ & $B_{5}$ & $B_{6}$ & $B_{7}$ \\
\hline \multirow{4}{*}{$\tilde{x}$} & $A_{1}$ & -15 & -15 & -15 & -10 & -10 & -5 & 0 \\
\cline { 2 - 9 } & $A_{2}$ & -15 & -15 & -10 & -10 & -5 & 0 & 5 \\
\cline { 2 - 9 } & $A_{3}$ & -15 & -10 & -10 & -5 & 0 & 5 & 10 \\
\cline { 2 - 9 } & $A_{4}$ & -10 & -10 & -5 & 0 & 5 & 10 & 10 \\
\cline { 2 - 9 } & $A_{5}$ & -10 & -5 & 0 & 5 & 10 & 10 & 15 \\
\cline { 2 - 9 } & $A_{6}$ & -5 & 0 & 5 & 10 & 10 & 15 & 15 \\
\cline { 2 - 9 } & $A_{7}$ & 0 & 5 & 10 & 10 & 15 & 15 & 15 \\
\hline
\end{tabular}

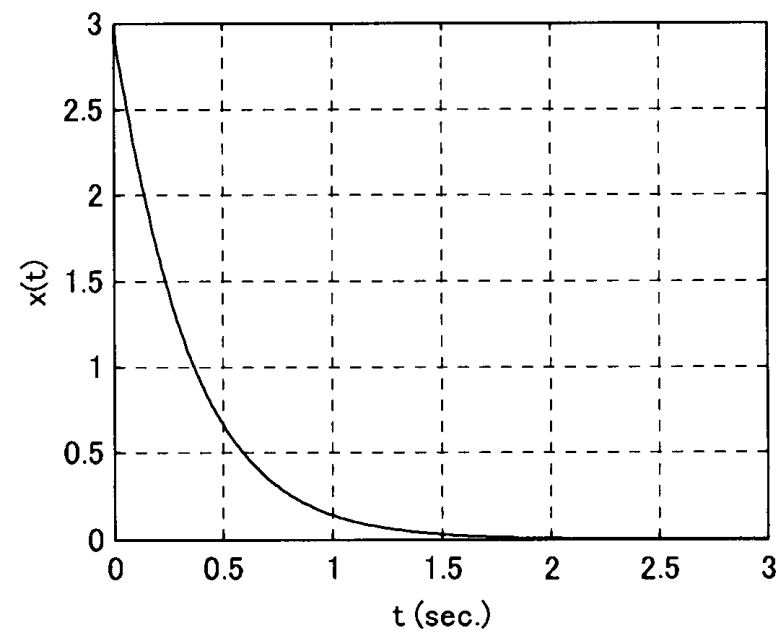

Figure 12: State evolution driven by the welltuned-fuzzy-controller

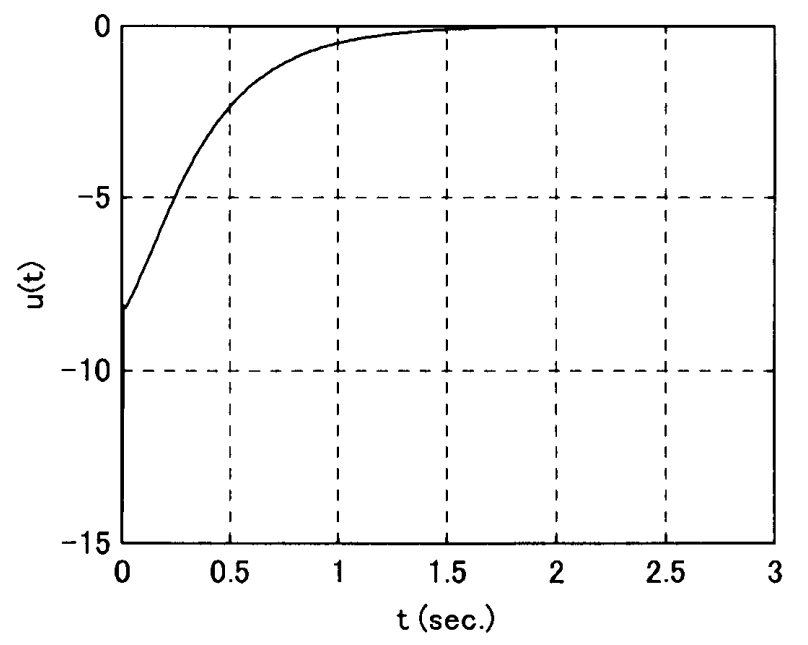

Figure 13: Controller moments obtained from the well-tuned-fuzzy-controller

clearly, is the best. We should note that in order to make all the results comparable, all the relevant coefficients are set same.

In addition, to prevent the chattering in the con- 


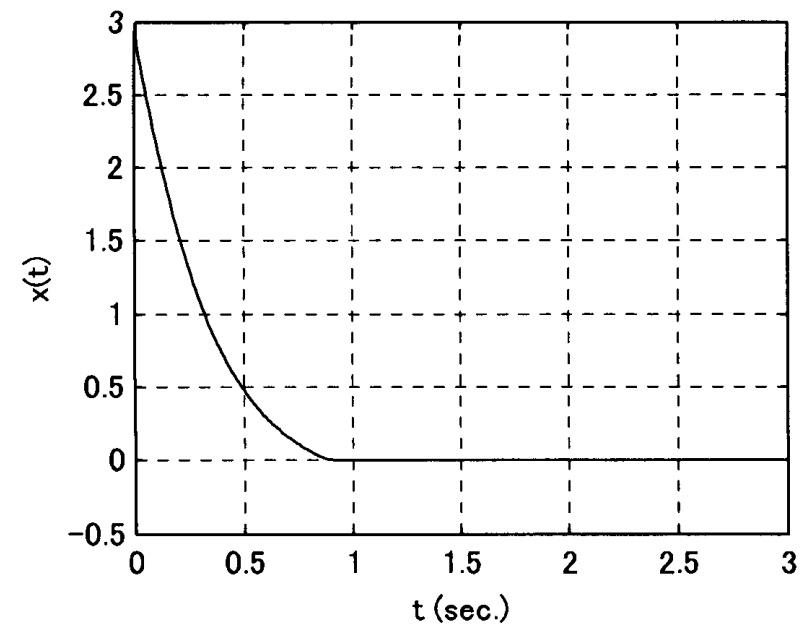

Figure 14: State evolution driven by the controller including the well-tuned-fuzzycontroller

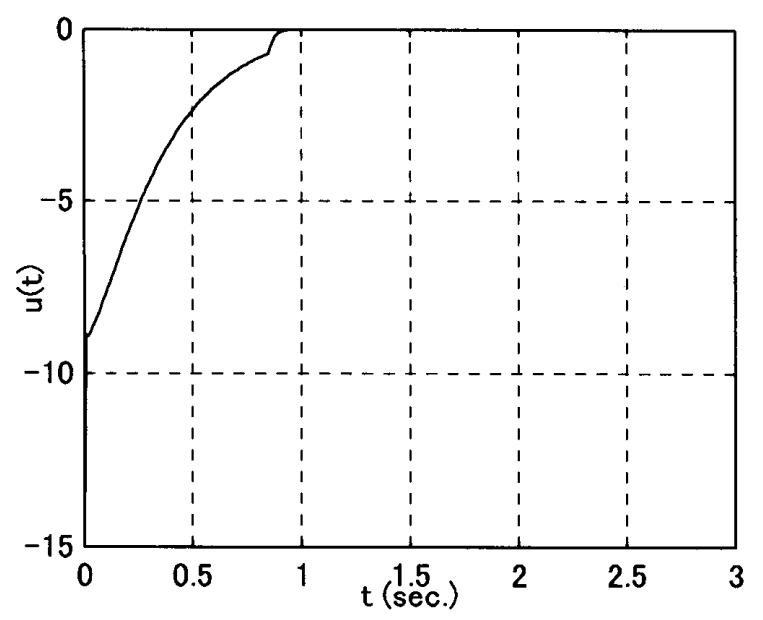

Figure 15: Controller moments including the well -tuned-fuzzy-controller

trol input because of the sign function $\operatorname{sgn}(s)$, in all the simulations conducted above we adopted a saturation function sat $(s / \phi)$ where $\phi=0.02$ is a little constant instead of $\operatorname{sgn}(s)$, consequently, the amount of $s$ is replaced by $s_{\phi}=s-\phi \operatorname{sgn}(s / \phi)$. Of course, the stability of the control system can be assured, and the analysis can be performed in the same manner as in subsection $3.2[8]$.

\section{CONCLUSION}

Directly employing the experienced experts' knowledge about the control process is the most important reason why fuzzy control has been greatly welcomed practically and theoretically for more than two decades. However, in most cases the adaptive fuzzy control systems proposed so far laid stress on system stabilities via the relevant parameters' tuning, rather than the involvement of experts' knowledge. In this paper, we gave details showing how to develop an adaptive control system in which experts' knowledge is taken into account wherever possible. Also, computer simulations confirmed the approach proposed in this paper.

\section{References}

[1] C.-Y. Su \& Y. Stepanenko, "Adaptive control of a class of nonlinear systems with fuzzy logic," IEEE Trans. on Fuzzy Systems, vol. 2, pp. 258-294, 1994.

[2] M. M. Polycarpou, "Stable adaptive neural control scheme for nonlinear systems," IEEE Trans. on Automatic Control, vol. 41, no. 3, pp. 447-451, 1996.

[ 3 ] M. M. Polycarpou \& M. J. Mears, "Stable adaptive tracking of uncertain systems using nonlinear parametrized on-line approximators," Int. J. Control, vol. 73, no. 3, pp. 363-384, 1998.

[4] H. Han, C.-Y. Su \& Y. Stepanenko, "Adaptive control of a class of nonlinear systems with nonlinearly parameterized fuzzy approximators," IEEE Trans. on Fuzzy Systems, vol. 9, no. 2, pp. 315-323, 2001.

[5] S. Tong \& H.-X. Li, "Fuzzy adaptive slidingmode control for MIMO nonlinear systems," IEEE Trans. on Fuzzy Systems, vol. 11, no. 3, pp. 354-360, 2003.

[6] L.-X. Wang \& J.M. Mendal, "Fuzzy basis function, universal approximation, and orthogonal least square learning," IEEE Trans. on Nerual networks, vol. 3, pp. 807-814, 1992.

[7] J.-J. Slotine, "Sliding controller design for nonlinear systems," Int. J. Control, vol. 40, no. 2, pp. 421-434, 1984.

[ 8 ] R. M. Sanner \& J.-J. Slotine, "Gaussian network for direct adaptive control," IEEE Trans. on Neural Networks, vol. 3, no. 6, pp.837-863, 1992.

[9] R. M. Sanner \& J.-J. E. Slotine, "Gaussian networks for direct adaptive control," IEEE Trans. on Neural networks, vol. 3, no. 6, pp.837-863, 1992.

(Received Sep 5, 2003)

(Accepted Dec. 12, 2003)

[Contact Address]

School of Business, Hiroshima Prefectural University 562 Nanatsuka, Shobara-shi, Hiroshima 727-0023, Japan TEL:08247-4-1706

FAX:08247-4-1706

E-mail : hhan@bus.hiroshima-pu.ac.jp 
Information about Author

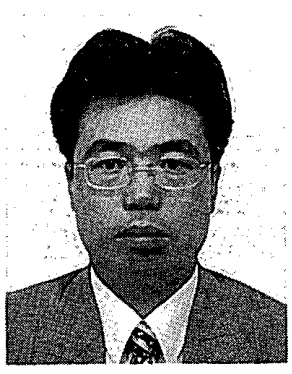

Hugang Han [member]

He received $\mathrm{Ph} . \mathrm{D}$. degree in electric engineering form Kyushu Institute of Technology in 1997.

From 1992 to 1994 he was with the Department of Automation at Northeast China Institute of Electric Power Engineering. Since 1997, he has been at Hiroshima Prefectural University where he is currently an Associate Professor of the Department of Management and Information Sciences. Dr. Han's main research interests are in fuzzy control, adaptive control and information extraction.

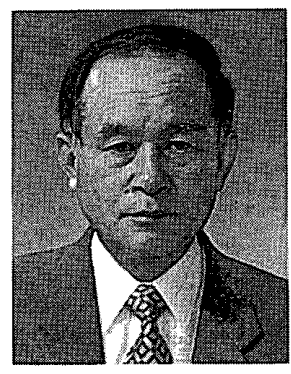

Shuta Murakami [member]

He received Ph.D. degree in control engineering from Tokyo Institute of Technology in 1969.

Since 1969, he has been with the Kyushu Institute of Technology, where he was a Lecturer with the Department of Control Engineering from 1969 to 1970, an Associate Professor from 1970 to 1984 . Since 1984, he has been a Professor with the Department of Computer Science. He is currently a member of the Board of Trustees of the Kyushu Institute of Technology. He has been a Fellow of the Operation Research Society of Japan since 1997. Dr. Murakami's main research interests are in fuzzy control, fuzzy modeling, fuzzy decision-making and so on.

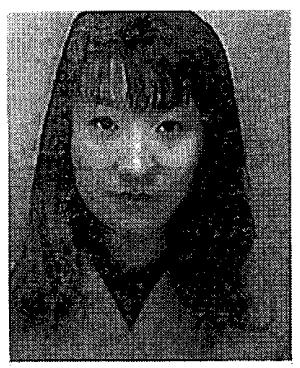

Hiroko Kawabata [non-member]

She received the MBA Degree in Management Information Theory from Graduate School of the Hiroshima Prefectural University, Japan in 1997.

From 1997 to 2002 she was a Lecturer of the Sannou Junior College concerning upon management theory. Since 2001, she has been a Lecturer of the Daiichikeizai University on multimedia theory.

Ms. Hiroko Kawabata's main academic interests are in fuzzy control, robust control, multimedia system. 